\title{
Trends of Piezoresistive Sensor Technology
}

Krause, Peter / Michels, Hendrik / Varma, Gyaneshwar

First Sensor Technology GmbH

Carl-Scheele-Str. 16

12489 Berlin, Germany

Pressure and temperature are the most frequently measured physical values. Pressure sensors are present in all kinds of daily applications. The most important are automotive, industrial, medical and household applications.

Today several principles are applied for pressure measurement and most principles measure a deflection of a membrane. Table 1 gives an overview of today's most important pressure sensor technologies and its typical pressure ranges.

\begin{tabular}{|l|l|l|}
\hline Diaphragm & Principle & Pressure range $\left[\mathrm{bar}=\mathbf{1 0}^{\mathbf{5}} \mathbf{P a}\right]$ \\
\hline Silicon & Piezoresistive & $0,005 \ldots 1.000$ \\
\hline Poly-Si & Capacitive & $0,5 \ldots 10$ \\
\hline \multirow{2}{*}{ Ceramic } & Capacitive & $0,001 \ldots 300$ \\
\cline { 2 - 3 } & Thick-/Thin film resistive & $0,1 \ldots 300$ \\
\hline \multirow{2}{*}{ Steel } & Thin film (metal or poly Si) & $1 \ldots .5000$ \\
\cline { 2 - 3 } & Microfused strain gage & $30 \ldots 2.000$ \\
\hline
\end{tabular}

Table 1: The most important pressure measurement technologies and its typical pressure ranges

The presentation discusses the current technological status of piezoresistive pressure sensors and its future development along the value chain. However there are conclusions which can be applied for other measurement principles.

The different measurement principles have specific advantages and disadvantages. The tables bellow gives an overview of the main advantages of piezoresistive pressure sensors:

\begin{tabular}{|lll|}
\hline Single-crystalline Silicon & $\rightarrow$ & ideal mechanical properties \\
& $\rightarrow$ & harsh environments applicable \\
Piezoresistive principle & $\rightarrow$ & high signal (k-factor) \\
Semiconductor technology & $\rightarrow$ & most experienced technology \\
MEMS technology & $\rightarrow$ & high miniaturisation \\
Compensation & $\rightarrow$ & standard electronic available \\
\hline
\end{tabular}

Table 2: Advantages of piezoresistive pressure sensors 


\section{The Value Chain}

The value chain of piezoresistive pressure sensors can be divided into four stages:
Front End I
Standard semiconductor processes
Front End II
MEMS specific processes
First Level Packaging
Packaging of sensors dies, ASIC's, ASSP's and passive components
Second Level Packaging
Housing and calibration of the sensor

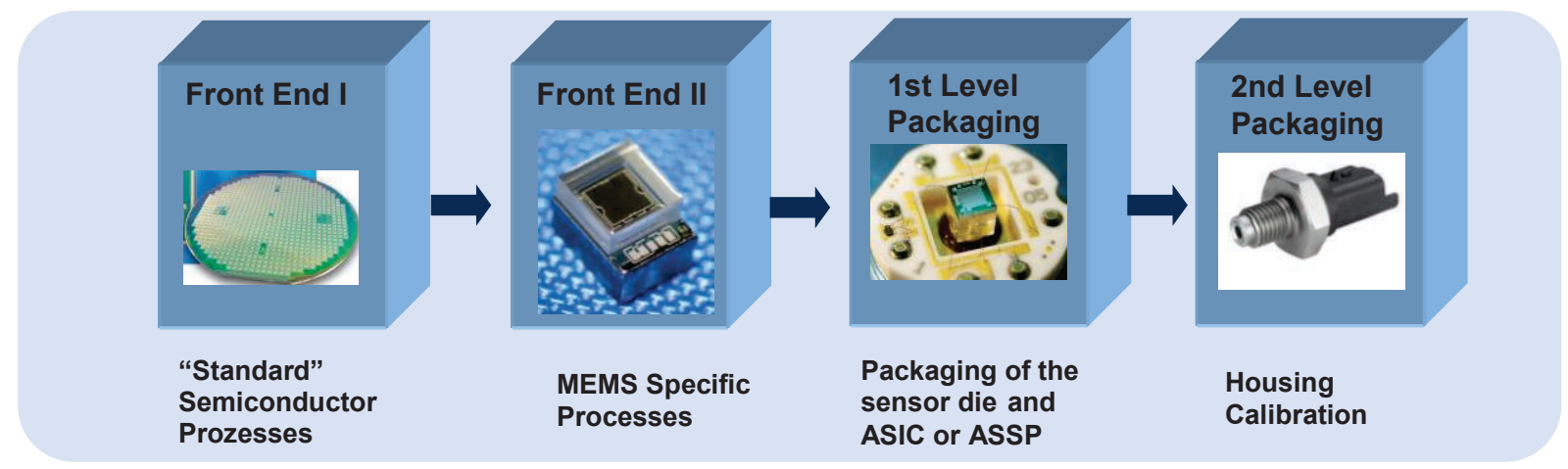

Picture 1: The value chain of piezoresistive pressure sensors

The Front End I processes are manufactured with standard semiconductor machines. For the sensor production different semiconductor processes like lithography and implantation are necessary. The biggest challenges are optimal utilization of the production capacity and the design of efficient processes. A high utilization of the production capacities can be achieved with contract manufacturing for many different customers or the production of several sensor types for one large company.

The Front End II processes (MEMS processes like membrane etching, wafer bonding or dicing) are technically not completely compatible to semiconductor processes. The development of compatible processes (e.g. dry etching of the membrane) has though developed significantly in the last years. It is the goal to use standard technologies for sensor production like TSV (through silicon vias).

To participate of the shift of the value creation, more and more FABs are offering now also MEMS processes. The requirements of theses sensors are usually low. In this case customers of pressure sensor dies and modules need profound know how, because the FABs are delivering the production service and not products and product support. The problem is that the stability of the piezoresistive sensor dies depends on the semiconductor and MEMS processes, but it can only be measured in complete sensors modules.

For the qualification and testing of the sensors dies a deep inside into the influences of the packaging and applications is necessary. Packaging can cause high mechanical stress on the sense die. This stress often leads to thermal hysteresis and signal drift. The manufacturer of pressure sensors dies has thus ideally a detailed know how of packaging and the various applications.

On the one hand it is the goal to use standard sense dies to have a cost efficient sensor solution. On the other hand innovative solutions are demanding application specific sensor dies. Every user should therefore know which production philosophy is necessary for the specific sensor application.

The First Level Packaging (PCB, ceramic board, sensor die, ASIC or ASSP) has a significant influence on the cost and performance of the sensor. It connects the sensor and ASIC with the carries and is normally done with standard equipment. The sensor die, the carrier material and the glue have a strong influence on the sensor performance and robustness. Many applications demand robust glue 
(vacuum sealed and resistant to aggressive media). However these glues are typically hard and have high demands regarding the mechanical stress on the pressure sensor die. To improve the robustness of the sensor thicker glass backplates can for instance be applied. This reduces the stress on the sensor itself.

The Second Level Packaging comprises the attachment of the connectors (pressure, electrical) and the calibration of the pressure sensor module. The calibration of the sensor has a strong impact on the accuracy and the costs of the sensor. Therefore it is done both in PCB panels or cycle calibrators. Trimming and hybrid calibration is still quiet common in industrial applications, though the trend shows that more ASIC's and ASSP's are used. Many products are using standard calibration. But the future trend demands specific calibration depending on the customized application.

\section{Trends}

The assessment of the sensor trends is based on experiences of the authors in the pressure sensor industry and the study "Sensor Trends 2014" of the AMA (association of the sensor industry in Germany).
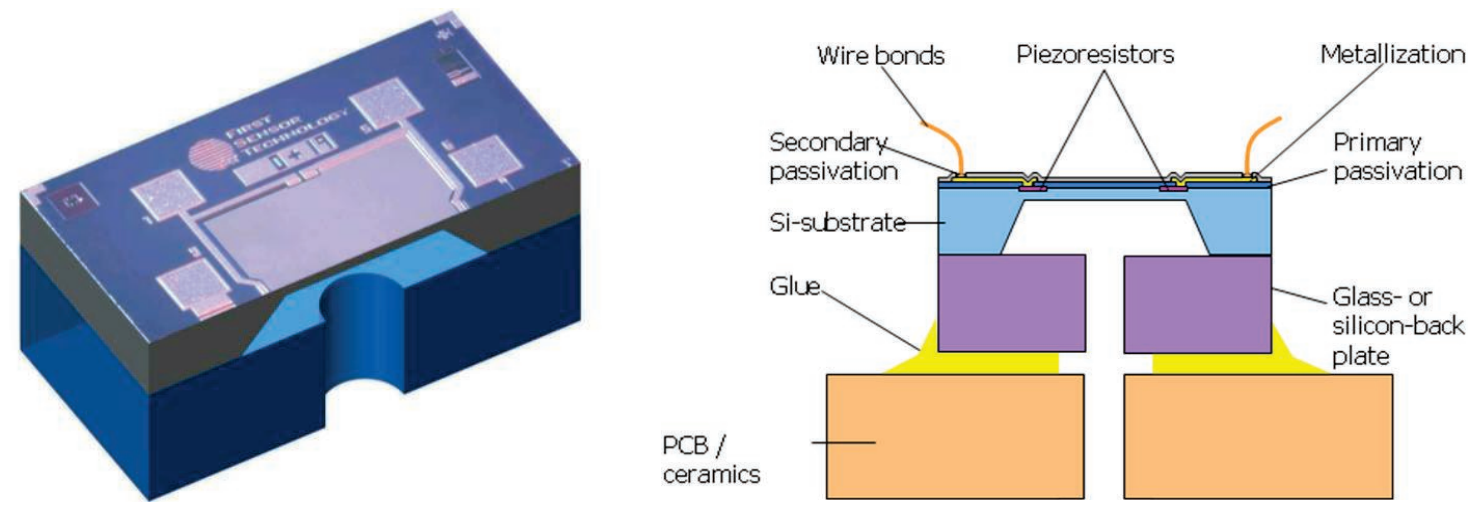

Picture 2: Structural drawing of a piezoresistive pressure sensor die

\section{Further Miniaturization}

The miniaturization is driven by cost pressure and geometrical demands. For piezoresistive pressure sensors most developments are done in the field of sense dies and packing. Today small elements have a edge length of $1.8 \mathrm{~mm}$, in the future sensors with edge length of $0,5 \mathrm{~mm}$ are to be expected. This small size is limited for absolute pressure sensors, because the reduction of the sensor size is also limiting the adhesive force of the glue.

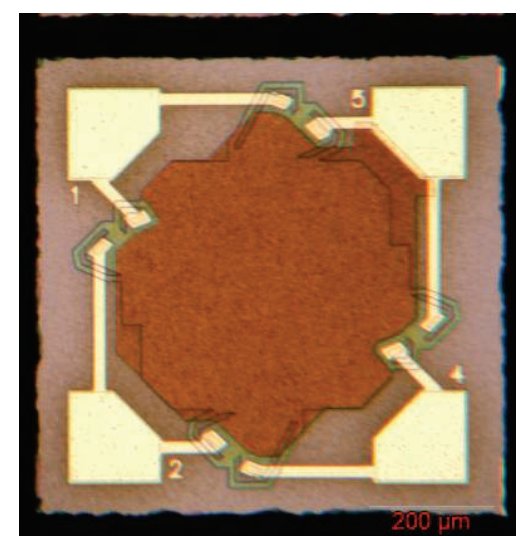

Picture 3: First Sensor Technology miniature piezoresistive pressure sensor die (edge length $0.75 \mathrm{~mm}$ ) 
The reduction of the size is possible due to the application of new technologies like DRIE (deep reactive-ion etching). Also the thinning of wafers is a way of miniaturization. However this is only used for special application like intraocular pressure.

The packaging allows smaller products due to new processes like MID (molded interconnected device) or flexible PCBs. In this field many application specific solutions are to be expected.

Miniaturization is also possible because of combined sensors, which not only reduce the number of necessary sense elements, but also the necessary packaging space.

\section{Reduction of Production Costs}

The cost reduction is the driving force in the pressure sensors market. This is achieved by more cost efficient sensors and the integration of new features and function for the same costs.

Within the value chain the following ways of cost reduction are possible:

- More functionalities on one sensor element (Front End I) or on one PCB ( $1^{\text {st }}$ Level Packing).

- Optimization of calibration processes $\left(2^{\text {nd }}\right.$ Level Packing $)$

- Miniaturization in all fields (e.g. Frond End I ASE, interlayer connections, reduction of the ASICs on its core function, MID)

- Standardization (e.g. design, electronic, interface)

\section{Increasing Intelligence}

Many sensor manufacturers add additional functions to their sensors. This is often done in connection with digital signals (e.g. to be able to transfer several signal over one electrical interface). However the analog interface will still remain an important interface for the next ten years.

As a result we see these trends:

- Digital signal processing

- Fail safe function (redundant system)

- Self calibration

- On-board diagnosis system

- Single chip solution

- Integration of multi sensor / embedded systems

\section{Reduced Measurement Inaccuracies}

The reduction of the measurement inaccuracies is a general trend in the pressure measurement technology. Even large volume applications are today demanding high accuracies.

The accuracy of pressure sensors is influenced by all steps of the value chain. The costs of a sensor depend on the accuracy, thus it is important to know which accuracy the specific applications really demands.

Generally one can say that $1 / 3$ of the inaccuracies comes from the sensor die, 1/3 from the packaging and $1 / 3$ from the calibration.

Regarding the measurement inaccuracies we can find the following trends:

- Development of more robust sensor elements (mechanically and electrically)

- New packaging technologies (e.g. to reduce the mechanical stress)

- Utilization of high performance sensor dies with cost efficient packaging and calibration 

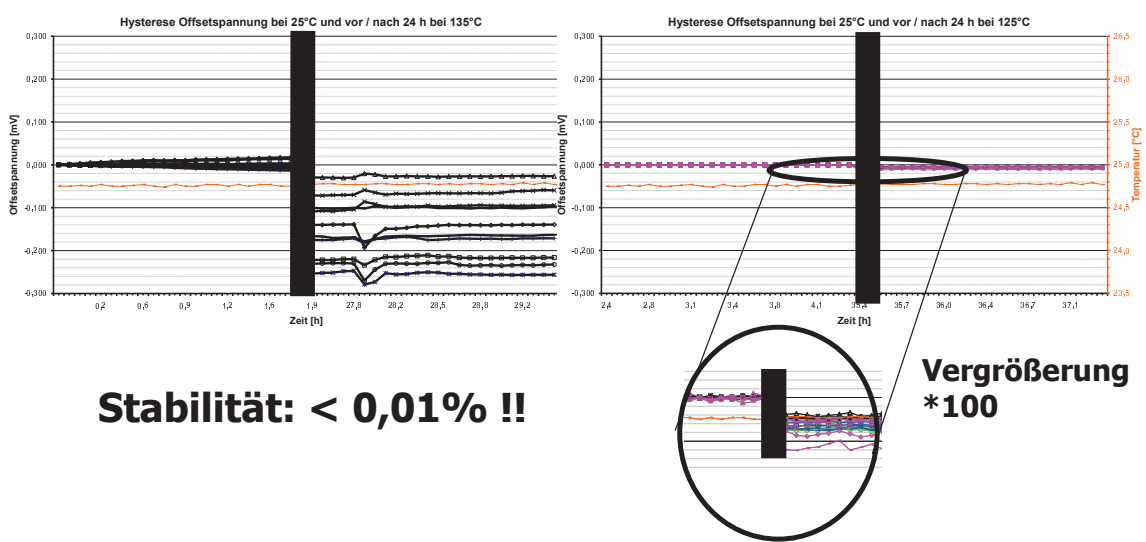

Picture 4: Stability of the FST STARe-Technology compared to the performance 10 years ago

\section{Increasing Demands of Senor Quality and Reliability}

High reliability of the pressure sensors is achieved through improvements in all stages of the value chain. This is ensured by frequent test during the whole production process. Test structures are used in the Front End I process. In the Front End II process 100\% tests during several process steps (e.g. membrane measurement, optical and electrical inspection) are necessary. A $100 \%$ end test after the calibration is today standard. Increasingly more effort is put into the qualification of sensors. The recommendation for "Robustness Validation" tries to apply the requirements of the AECQ100 that can normally not be applied for sensors.

\section{Wireless Sensors and Reduced Energy Consumption}

Wireless transmission of measurement signals is highly demanded in modern applications. For wireless measurement low energy sensor elements are necessary. Capacitive sensors are often used for low energy applications, but advanced resistive principles are today consuming also little energy. Intelligent control technologies reduce the energy consumption. ASIC's and microprocessors are today using lower supply voltages $(1,8 \mathrm{~V})$ and therefore demanding compatible sensor dies.

\section{Expansion of the Pressure Range}

New technologies allow now the application of piezoresistive pressure sensors for both very low and very high pressures. Low pressure ranges can be measured with an improved center boss technology (reduced dependence of the position of the sensor). In the low pressure range now pressures below 10 mbar are covered with piezoresistive pressure sensors. On the other end of the scale piezoresistive sensors can today be used for pressures of 5000 bar and more. These high pressure ranges can be measured with a combination of a steel membrane and a piezoresistive sensor die.
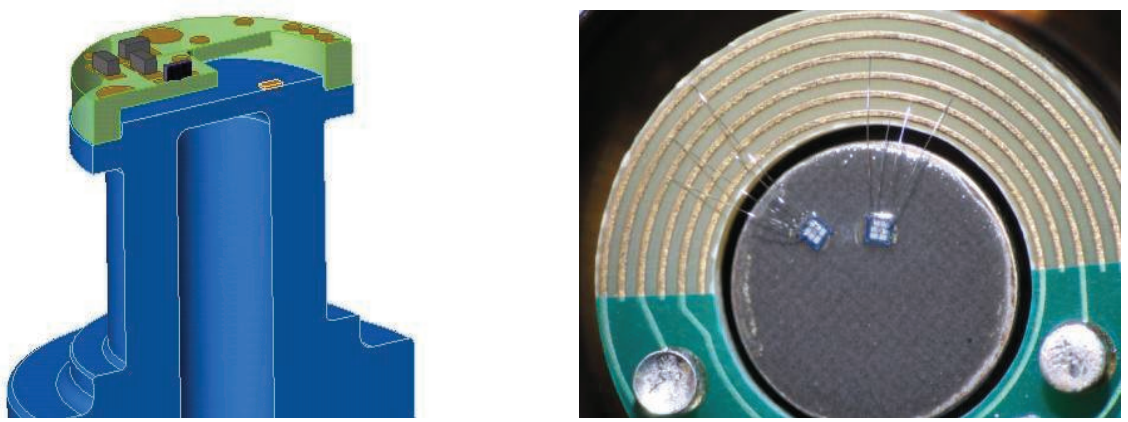

Picture 5: High pressure technology of FST, the T-Bridge on a steel membrane 


\section{Expansion of the Temperature Range}

A broader temperature range with up to $150^{\circ} \mathrm{C}$ can now be covered with standard semiconductor technologies. The SOI technology makes also temperature over $200^{\circ} \mathrm{C}$ possible. In SOI technology the epitaxial deposition of silicon is done on the insulating material (buried $\mathrm{SiO}_{2}$ ) to reduce the temperature depended parameters. However this technology is more expensive and covers only a small part of the pressure sensor market. 